\title{
Metabolic Biomarker-Based BRAFV600 Mutation Association and Prediction in Melanoma
}

\author{
Hanna Saadani*1, Bernies van der Hiel*1, Else A. Aalbersberg ${ }^{1}$, Ioannis Zavrakidis ${ }^{2}$, John B.A.G. Haanen ${ }^{3}$, \\ Otto S. Hoekstra ${ }^{4}$, Ronald Boellaard ${ }^{4}$, and Marcel P.M. Stokkel ${ }^{1}$ \\ ${ }^{I}$ Department of Nuclear Medicine, Netherlands Cancer Institute, Amsterdam, The Netherlands; ${ }^{2}$ Department of Epidemiology and \\ Biostatistics, Netherlands Cancer Institute, Amsterdam, The Netherlands; ${ }^{3}$ Department of Medical Oncology, Netherlands Cancer \\ Institute, Amsterdam, The Netherlands; and ${ }^{4}$ Department of Radiology and Nuclear Medicine, Amsterdam UMC, Vrije Universiteit \\ Amsterdam, Cancer Center Amsterdam, Amsterdam, The Netherlands
}

See an invited perspective on this article on page 1543.

The aim of this study was to associate and predict B-rapidly accelerated fibrosarcoma valine 600 (BRAFV600) mutation status with both conventional and radiomics ${ }^{18} \mathrm{~F}-\mathrm{FDG}$ PET/CT features, while exploring several methods of feature selection in melanoma radiomics. Methods: Seventy unresectable stage III-IV melanoma patients who underwent a baseline ${ }^{18} \mathrm{~F}-\mathrm{FDG}$ PET/CT scan were identified. Patients were assigned to the BRAFV600 group or BRAF wild-type group according to mutational status. ${ }^{18} \mathrm{~F}-\mathrm{FDG}$ uptake quantification was performed by semiautomatic lesion delineation. Four hundred eighty radiomics features and 4 conventional PET features $\left(S U V_{\max }\right.$, $\mathrm{SUV}_{\text {mean }}, \mathrm{SUV}_{\text {peak }}$, and total lesion glycolysis) were extracted per lesion. Six different methods of feature selection were implemented, and 10-fold cross-validated predictive models were built for each. Model performances were evaluated with areas under the curve (AUCs) for the receiver operating characteristic curves. Results: Thirty-five BRAFV600 mutated patients (100 lesions) and 35 BRAF wild-type patients (79 lesions) were analyzed. AUCs predicting the BRAFV600 mutation varied from 0.54 to 0.62 and were susceptible to feature selection method. The best AUCs were achieved by feature selection based on literature, a penalized binary logistic regression model, and random forest model. No significant difference was found between the BRAFV600 and BRAF wild-type group in conventional PET features or predictive value. Conclusion: BRAFV600 mutation status is not associated with, nor can it be predicted with, conventional PET features, whereas radiomics features were of low predictive value (AUC $=0.62$ ). We showed feature selection methods to influence predictive model performance, describing and evaluating 6 unique methods. Detecting BRAFV600 status in melanoma based on ${ }^{18} \mathrm{~F}-\mathrm{FDG}$ PET/CT alone does not yet provide clinically relevant knowledge.

Key Words: ${ }^{18} \mathrm{~F}-\mathrm{FDG}$ PET/CT; melanoma; BRAF; radiomics

J Nucl Med 2019; 60:1545-1552

DOI: 10.2967/jnumed.119.228312

\footnotetext{
Received Mar. 6, 2019; revision accepted Aug. 5, 2019.

For correspondence or reprints contact: Bernies van der Hiel, Department of Nuclear Medicine, Netherlands Cancer Institute, Plesmanlaan 121,1066CX Amsterdam, The Netherlands.

E-mail: b.vd.hiel@nki.nl

${ }^{*}$ Contributed equally to this work.

Published online Sep. 3, 2019.

COPYRIGHT (C) 2019 by the Society of Nuclear Medicine and Molecular Imaging.
}

$\mathbf{M}$ elanoma is the most aggressive and deadliest type of skin cancer. It belongs to one of the most common cancers worldwide, and its incidence is rising (1). Approximately 50\% of melanomas contain a B-rapidly accelerated fibrosarcoma (BRAF) mutation, of which the most common is BRAF valine 600 (BRAFV600). The BRAF mutation causes constitutive activation of the mitogen-activated protein kinase pathway, which drives tumor growth, cell proliferation, and metastasis $(2,3)$. BRAF mutation determination is the decisive factor for commencing first-line BRAF inhibition in metastatic melanoma: a pivotal moment in clinical decision making. Similarly crucial are staging, restaging, and follow-up in metastatic melanoma, which is reliant on ${ }^{18} \mathrm{~F}-\mathrm{FDG}$ PET/CT imaging (2). BRAF mutation prediction based on PET/CT could combine these 2 clinical crossroads. In melanoma, the potential of PET/CT-based prediction has been mainly explored in conventional PET features and visual assessments, whereas current developments in precision medicine increase the need for in-depth tumor characterization (4). Unlocking this information traditionally would necessitate extensive pathologic research of all individual tumor lesions: an invasive manner to determine each lesion's biologic constitution. Radiomics analysis provides the ability to extract a possibly infinite amount of quantitative imaging features representing tumor characteristics, circumventing these negatives while venturing beyond. In colorectal and lung cancer, several PET radiomics features have been associated with disease-specific mutations, with area under the receiver operating characteristic curve (AUC) of their mutation prediction models ranging between 0.33 and 0.79 (5-7).

Between performing a scan and model building, several steps are required in radiomics analysis. An important challenge in the current state of radiomics analysis is the small patient sample size combined with infinite possibilities for image feature extraction. Feature selection and reduction is therefore necessary to avoid false-positive outcomes. Within previous research in other cancer types, a large variety of methods prevail, from preselection and prereduction to statistical corrections after analysis (8). Since feature selection impacts which features are ultimately chosen for a prognostic or diagnostic model, a uniform approach would be ideal. No previous PET radiomics research in melanoma is available; therefore, it is unclear how these methods would perform in melanoma. The aim of this study was to associate and predict BRAFV600 mutation status with both conventional and radiomics ${ }^{18}$ F-FDG PET/CT features, while exploring several methods of feature selection in melanoma radiomics. 


\section{MATERIALS AND METHODS}

\section{Patients}

Melanoma patients receiving BRAF inhibitors or immunotherapy were searched from June 2014 until March 2017. Eligible patients were the first consecutive 35 BRAFV600 mutated or 35 BRAF wild-type patients. Inclusion criteria were unresectable stage III-IV pathologyconfirmed melanoma, known BRAFV600 mutation status, and measurable disease on baseline ${ }^{18} \mathrm{~F}-\mathrm{FDG}$ PET/CT. BRAFV600 mutation status was determined by mutation analysis. Patients with rat sarcoma (RAS) mutations and systemic therapy (chemotherapy, BRAF inhibitors, or immunotherapy) 3 mo before baseline PET/CT were excluded (Fig. 1 provides the other exclusion criteria). Radiotherapy and any invasive local intervention within 3 mo before baseline PET/CT were registered. The institutional review board approved this retrospective study, and the requirement to obtain informed consent was waived.

\section{${ }^{18}$ F-FDG PET/CT Imaging}

${ }^{18} \mathrm{~F}$-FDG PET/CT imaging was performed $60 \mathrm{~min}$ after intravenous injection of $190-260 \mathrm{MBq}$ of ${ }^{18} \mathrm{~F}-\mathrm{FDG}$ after fasting for at least $6 \mathrm{~h}$.

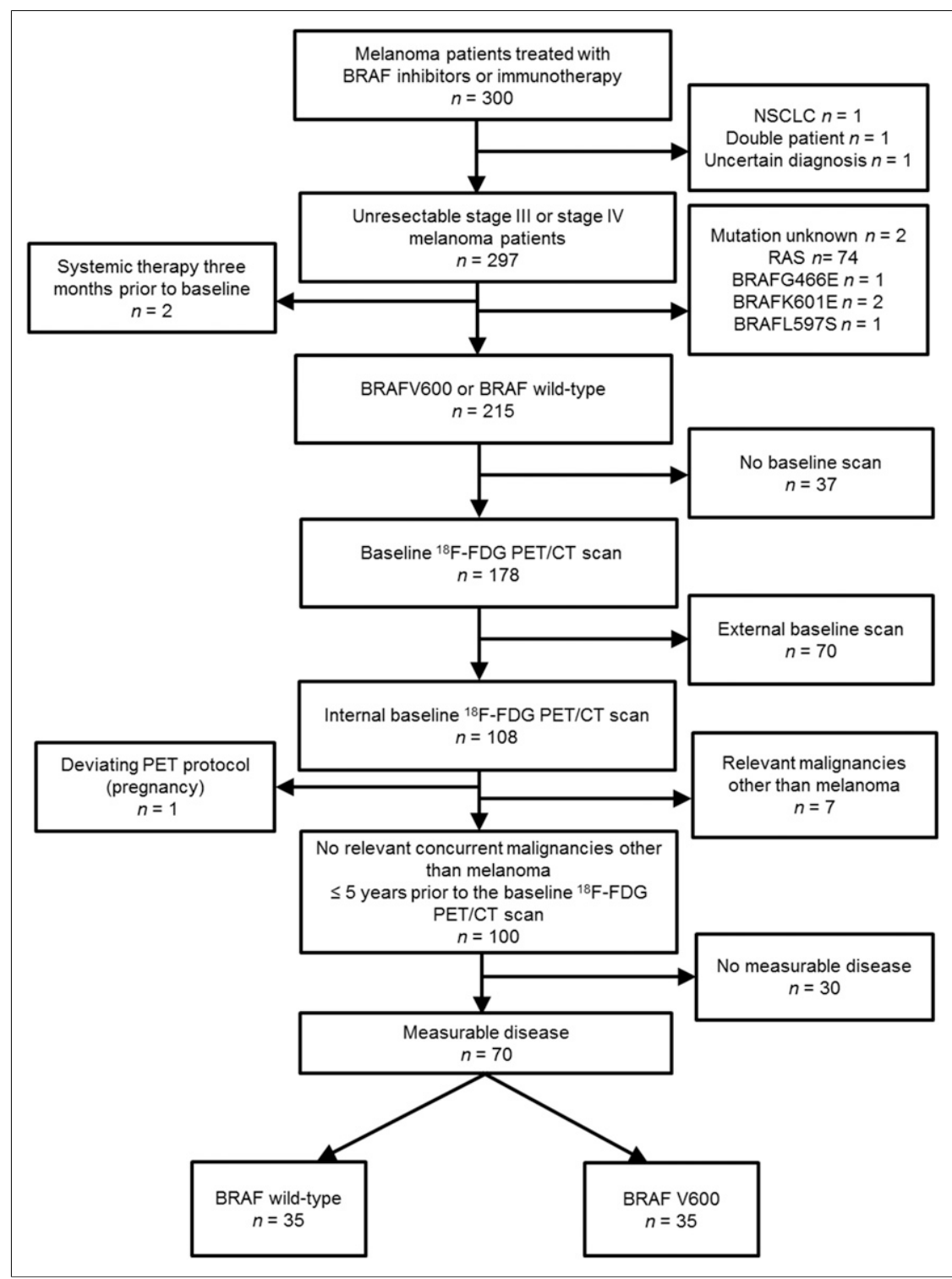

FIGURE 1. Patient selection flowchart.
Patients' injected dose was body mass index-based, and blood glucose levels before injection were required to be less than $200 \mathrm{mg} / \mathrm{dL}$. Patients were scanned on a cross-calibrated (with calibration phantoms) Phillips Gemini TF time-of-flight 16 or Phillips Gemini TF big-bore PET/CT scanner, with 1-3 min per bed position. The systems were from the same vendor and have the same type of acquisition and image reconstruction methods, and the same settings were used. PET images were reconstructed using BLOB ordered-subsets time-of-flight with 3 iterations, 31 subsets, no filter, voxel size of $4 \times 4 \times 4 \mathrm{~mm}$, slice thickness of $4 \mathrm{~mm}$, and image matrix of $144 \times 144$ pixels. This resulted in a postreconstruction resolution at $1 \mathrm{~cm}$ after line-of-response construction of $4.3 \mathrm{~mm}$. Low-dose CT scans were performed for attenuation correction and anatomic correlation (40 mAs, $140 \mathrm{keV}, 5-\mathrm{mm}$ slices).

\section{Image and Quantitative PET/CT Analysis}

Tumor lesion size was measured in the axial plane on the concurrent lowdose CT scan or on a baseline diagnostic CT or MRI scan acquired within 1 mo from the baseline PET/CT using OsiriX MD (Pixmeo Sarl, version 7.0.3). Measurable disease was defined by lesions of at least $2 \mathrm{~cm}$ or (if the tumor was indiscernible on CT) an equivalent metabolic active tumor volume (MATV) of at least $4.2 \mathrm{~cm}^{3}$, in line with PERCIST 1.0 to avoid partial-volume effects (9). Of the measurable lesions in each patient, the 3 lesions with the highest $\mathrm{SUV}_{\text {max }}$ per organ were considered target lesions. Target lesions were delineated by a threshold of $50 \%$ of the $\mathrm{SUV}_{\max }$ without background correction (9). The specified body organ regions were according to melanoma's metastasis pattern: lymph nodes, lung, liver, bone, subcutaneous, intramuscular, and other. Lesions with prior radiotherapy were excluded as target lesions. Quantitative PET/CT analysis was performed using in-house software tools (Fig. 2) $(10,11)$.

For radiomics analysis, 480 features were extracted, pertaining to morphology $(n=22)$, local intensity $(n=2)$, intensity-based statistics $(n=18)$, intensity-volume histogram $(n=6)$, intensity histogram $(n=24)$, and texture $(n=$ 408) (Supplemental Table 1; supplemental materials are available at http://jnm.snmjournals.org). Texture features were based on the gray level co-occurrence matrix (GLCM), gray level run length matrix (GLRLM), gray level size zone matrix (GLSZM), gray level distance zone matrix (GLDZM), neighborhood gray tone difference matrix (NGTDM), and neighboring gray level dependence matrix (NGLDM) with up to 8 matrix calculation methods. The features underwent $2 \times 2 \times 2 \mathrm{~mm}$ voxel resampling and discretization with a fixed bin size of 0.25 SUV (10). All image-processing and feature calculations conform with the image biomarker standardization initiative (12). Conventional PET features measured per lesion were MATV, $\mathrm{SUV}_{\text {max }}, \mathrm{SUV}_{\text {peak }}, \mathrm{SUV}_{\text {mean }}$, and total lesion glycolysis (TLG, defined as $\mathrm{SUV}_{\text {mean }} \times$ MATV). Uptake interval times, liver and blood pool $\mathrm{SUV}_{\text {mean }}$ were measured (13).

\section{Statistical Analysis}

A sample size calculation was performed combining a mean melanoma $\mathrm{SUV}_{\max }$ from 


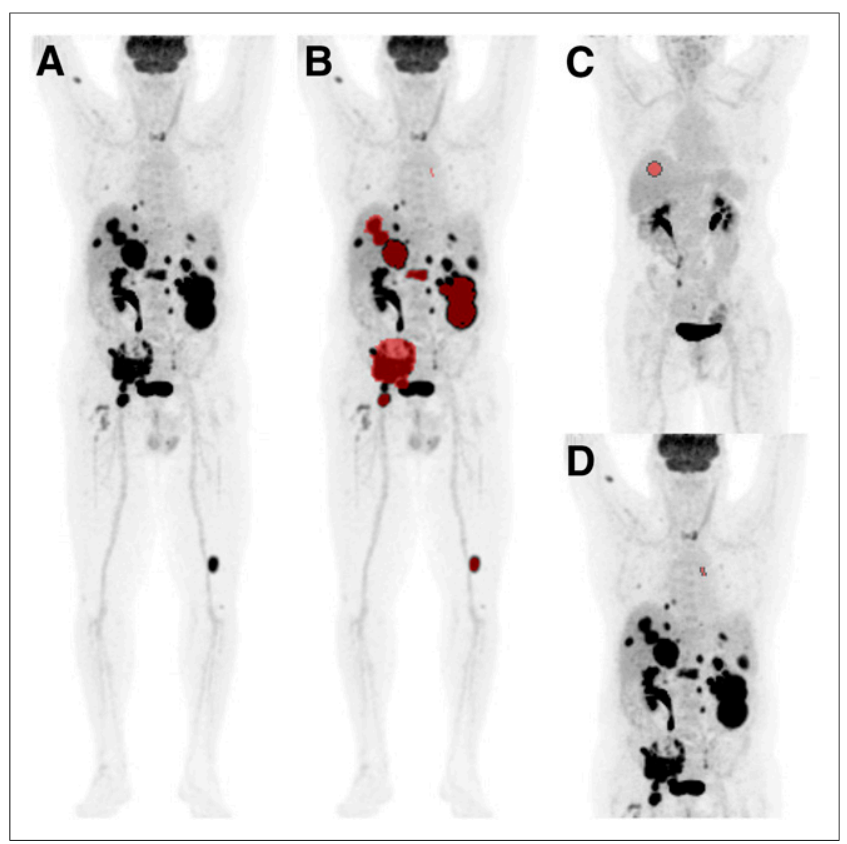

FIGURE 2. Anterior maximum-intensity-projections (A) with semiautomatic delineation of target lesions (B), liver (C), and blood pool (D).

prior studies (14) and an expected biologically relevant difference of at least $20 \%$ in $\mathrm{SUV}_{\max }$ between the mutated and nonmutated groups. This expected difference was based on a minimum biologically relevant SUV difference $(9,15)$. Assuming that mean $\mathrm{SUV}_{\max }$ in the BRAF wildtype group is 7.6 and $\mathrm{SD}$ of $\mathrm{SUV}_{\max }$ is 5.4 , with 60 patients and 3 lesions per patient, a difference between the 2 groups of at least $20 \%$ can be detected with a power of $80 \%$. Such a difference corresponds to a mean SUV $_{\text {max }}$ of at least 9.1 in the BRAFV600 group. These calculations are based on a mixed-effects model with the logarithm of $\mathrm{SUV}_{\text {max }}$ as the outcome. All continuous variables were assessed for normality combining visual (histograms) and statistical inspection (Shapiro-Wilk test). Normally distributed patient characteristics were compared between BRAFV600 and BRAF wild-type groups with an independent-samples $t$ test. Not-normally distributed variables were log-transformed to achieve normality. If the transformation did not solve the distributional issue, a Mann-Whitney $U$ test was conducted. Categoric patient characteristics and target lesion distribution were compared between groups with a Pearson $\chi^{2}$ test or Fisher exact test.

Conventional PET features were compared between patient groups with mixed models, to account for multiple lesions from the same patient. The SUV metrics, TLG, MATV, and longest diameter were analyzed with linear mixed models, whereas prior local intervention was analyzed with generalized linear mixed models. Mutation status was used as a fixed effect, and patient was used as a random effect in the models. To facilitate interpretation of the mixed-model analysis, the original means instead of the log-transformed values were reported. To predict the BRAFV600 mutation with the conventional PET features, first the best binary logistic regression model of each feature and combination was determined, and then it was used for the final binary logistic regression and random forest (RF) model.

Six different methods for radiomics feature selection were applied: a correlation matrix of all features (method 1); a correlation matrix of all features with the conventional PET features $\mathrm{SUV}_{\text {peak }}$, MATV, and TLG (method 2); a principal-component analysis (PCA) (method 3); selection of features from prior studies (method 4); a penalized binary logistic regression analysis of all features (method 5); and an RF model of all features (method 6) (16). In method 1, correlation between any 2 features was calculated, and from highly correlated pairs (Spearman correlation $>0.75$ ) the one feature that had an average highest correlation with all other features was removed. Method 2 followed the same principle, except feature reduction was first based on all radiomics features with $S_{U V}$ peak, TLG, and MATV and then on the remainder of features. Method 3 began as method 1 did, but with a threshold Spearman correlation higher than 0.85 , considering its predictive nature. The residual features were standardized based on mean $=0$ and $\mathrm{SD}=1$ for entry into the PCA, a dimensionality reduction method. PCA, using mathematic projection, transforms the data into a set of orthogonal variables called principal components, which get ranked based on the data variance along them. The highest data variance is represented by the first principal component, whereas the subsequent ones achieve the highest possible variance orthogonal to the prior. From the first 10 principal components, the 20 most important features per each were extracted. Finally, the 20 most important features over all 10 principal components were determined. The number of features was based on the standard statistical practice of approximately 10 observations per radiomics feature in line with Collarino et al. and Chalkidou et al. (17,18). Since we had 179 lesions, approximately up to 20 features could be included in a model. In method 4, 10 features repeatedly reported as robust in prior studies $(10,19-23)$ were selected, focusing on the best test-retest repeatability. Method 5 was built with all radiomics features; regression coefficients were penalized using the elastic net regularization correcting for group effect (collinearity) and removing less relevant coefficients (24). Method 6 was construed from all radiomics features, without recursive feature elimination. RF is an ensemble method for building prediction models and is especially useful for high-dimensional data for which the number of features exceeds the number of observations. RF builds several decision trees and eventually averages the results. Here, 1,000 trees were used for predicting the mutation class, and 21 variables were randomly sampled at each split.

After feature selection methods $1-4$, the final features were inserted in a binary logistic regression model and RF model to predict the BRAFV600 mutation. All models were cross-validated via 10-fold cross-validation repeated 10 times, and their respective AUCs were composed. Statistical analysis was performed with SPSS (IBM, version 22.0) and R software (version 3.4.4) with, respectively, the nonlinear mixed effects and linear mixed effects 4 package for the linear mixed model and generalized linear mixed model $(25,26)$. Caret and RF packages were used for the RF models, and generalized linear models with lasso or elastic net regularization were used for the penalized binary logistic regression (27-29). A $P$ value of less than 0.05 was considered significant.

\section{RESULTS}

Seventy patients were identified, 35 patients per BRAFV600 or BRAF wild-type group (Fig. 1). Patient characteristics are listed in Table 1. Per patient, 1-10 target lesions were analyzed. The BRAFV600 and BRAF wild-type groups showed no statistically significant differences in SUV metrics, MATV, TLG, longest diameter, or prior local intervention (Table 2). SUV metrics and TLG stratified per organ region showed the same result (Supplemental Table 2). The liver and blood pool $\mathrm{SUV}_{\text {mean }}$ were tested for confounding, which was not found (data not shown). The best conventional PET features prediction model was achieved by inserting all 5 features: $\mathrm{SUV}_{\text {mean }}, \mathrm{SUV}_{\text {max }}, \mathrm{SUV}_{\text {peak }}$, TLG, and MATV (Fig. 3A; Supplemental Table 3A). Radiomics analysis was performed on 176 lesions (3 lesions from scans with a different voxel matrix were excluded). Four of the 480 radiomics 
TABLE 1

Patient Characteristics and Target Lesion Distribution

\begin{tabular}{|c|c|c|c|}
\hline Characteristic & BRAFV600, $n=35$ & BRAF wild-type, $n=35$ & $P$ \\
\hline Age (y) & $55.5(11.7)$ & $64.7(13.2)$ & $0.003^{\star}$ \\
\hline Sex & & & $0.631^{\dagger}$ \\
\hline Male & $18(51.4 \%)$ & $20(57.1 \%)$ & \\
\hline Female & $17(48.6 \%)$ & $15(42.9 \%)$ & \\
\hline Tumor type & & & $0.071^{\ddagger}$ \\
\hline Cutaneous & $32(91.4 \%)$ & $25(71.4 \%)$ & \\
\hline Mucosal & $1(2.9 \%)$ & $6(17.1 \%)$ & \\
\hline Unknown primary & $2(5.7 \%)$ & $4(11.4 \%)$ & \\
\hline AJCC stage, eighth edition & & & $1.000^{\ddagger}$ \\
\hline IIIB & $2(5.7 \%)$ & $2(5.7 \%)$ & \\
\hline IIIC & $2(5.7 \%)$ & $2(5.7 \%)$ & \\
\hline IV & $31(88.6 \%)$ & $31(88.6 \%)$ & \\
\hline Tumor load & & & $0.626^{\dagger}$ \\
\hline$<6$ lesions & $15(42.9 \%)$ & $13(37.1 \%)$ & \\
\hline$\geq 6$ lesions & $20(57.1 \%)$ & $22(62.9 \%)$ & \\
\hline \multicolumn{4}{|l|}{ Organ involvement } \\
\hline Lymph nodes & $26(74.3 \%)$ & $26(74.3 \%)$ & $1.000^{\dagger}$ \\
\hline Lung & $17(48.6 \%)$ & $19(54.3 \%)$ & $0.632^{\dagger}$ \\
\hline Liver & $14(40.0 \%)$ & $9(25.7 \%)$ & $0.203^{\dagger}$ \\
\hline Bone & $10(28.6 \%)$ & $11(31.4 \%)$ & $0.794^{\dagger}$ \\
\hline Subcutaneous & $15(42.9 \%)$ & $21(60.0 \%)$ & $0.151^{\dagger}$ \\
\hline Intramuscular & $10(28.6 \%)$ & $7(20.0 \%)$ & $0.403^{\dagger}$ \\
\hline Other & $24(68.6 \%)$ & $15(42.9 \%)$ & $0.030^{\dagger}$ \\
\hline \multicolumn{4}{|l|}{ Scan characteristics } \\
\hline Glucose (mmol/L) & 5.4 \% $(0.82)$ & $5.6(0.98)$ & $0.417^{\S}$ \\
\hline Net injected activity (MBq) & $194.7(21.4)$ & $203.5(26.6)$ & $0.155^{\S}$ \\
\hline Body weight $(\mathrm{kg})$ & $78.9(13.9)$ & $84.1(20.0)$ & $0.397^{\S}$ \\
\hline Uptake interval (min) & $68.1(9.0)$ & $64.9(8.5)$ & $0.107^{\S}$ \\
\hline Blood pool SUV mean & $1.67(0.26)$ & $1.84(0.27)$ & $0.006^{\star}$ \\
\hline Liver SUV $\mathrm{V}_{\text {mean }}$ & $2.40^{\| \prime}(0.40)$ & $2.74^{\#}(0.41)$ & $0.003^{*}$ \\
\hline \multicolumn{4}{|l|}{ Target lesion distribution } \\
\hline Total & 100 lesions & 79 lesions & \\
\hline Lymph nodes & 37 & 32 & $0.162^{\ddagger}$ \\
\hline Lung & 5 & 13 & \\
\hline Liver & 17 & 10 & \\
\hline Bone & 13 & 6 & \\
\hline Subcutaneous & 11 & 8 & \\
\hline Intramuscular & 2 & 0 & \\
\hline Other & 15 & 10 & \\
\hline
\end{tabular}

*Independent-samples $t$ test.

${ }^{\dagger}$ Pearson $\chi^{2}$ test.

${ }^{\ddagger}$ Fisher exact test.

I $n=32$ (3 missing values).

${ }^{\S}$ Mann-Whitney $U$ test

$\|_{n}=26$.

${ }^{\#} n=31$.

AJCC $=$ American Joint Committee on Cancer.

Data are displayed as mean followed by SD in parentheses or as $n$ followed by percentage in parentheses. Data may not add up to $100 \%$ due to rounding.

1548 The Journal of Nuclear Medicine • Vol. 60 • No. 11 • November 2019 
TABLE 2

Conventional PET Features and BRAFV600 Mutation Association

\begin{tabular}{|c|c|c|c|}
\hline Parameter & BRAFV600, $n=35$ & BRAF wild-type, $n=35$ & $P$ \\
\hline SUV $_{\max }$ & $13.3(5.4)$ & $15.1(11.2)$ & $0.791^{*}$ \\
\hline SUV $_{\text {peak }}$ & $10.7(4.6)$ & $11.8(8.9)$ & $0.979^{\star}$ \\
\hline $\mathrm{SUV}_{\text {mean }}$ & $8.9(3.6)$ & $10.1(7.6)$ & $0.768^{*}$ \\
\hline TLG $_{\text {target }}{ }^{\dagger}$ & $191.0(360.3)$ & $126.4(183.6)$ & $0.185^{\star}$ \\
\hline $\operatorname{MATV}\left(\mathrm{cm}^{3}\right)$ & $18.8(33.0)$ & $14.1(22.4)$ & $0.093^{*}$ \\
\hline Size $(\mathrm{mm})$ & $37.8^{\ddagger}(20.6)$ & $32.8^{\text {Il }}(16.5)$ & $0.150^{*}$ \\
\hline \multicolumn{4}{|c|}{ Local intervention before baseline PET/CT per target lesion } \\
\hline Yes & $25(25 \%)$ & $14(17.7 \%)$ & $0.240^{\S}$ \\
\hline No & 75 (75\%) & 65 (82.3\%) & \\
\hline \multicolumn{4}{|c|}{$\begin{array}{l}{ }^{*} \text { Linear mixed model. } \\
{ }^{\dagger} T L G{ }_{\text {target }}: \text { TLG based on target lesions only. } \\
{ }^{{ }^{+}} n=25,84 \text { lesions. } \\
{ }^{I_{n}} n=24,68 \text { lesions. } \\
{ }^{\S} \text { Generalized linear mixed model. } \\
\text { Data are displayed as mean followed by SD }\end{array}$} \\
\hline
\end{tabular}

features maintained a value of 1 for all lesions and were removed, which were from the neighboring gray level dependence matrix: dependence count percentage (averaged over 2D slices and directions), dependence count percentage (3D volume), dependence count percentage (2D merged over all slices), and from the intensity histogram: minimum. After removal of high correlations in method 1, 23 features remained from the 476 with AUCs of 0.55 (Fig. 3B; Supplemental Table 3B). Method 2 resulted in 26 features after removal of high correlations with AUCs of 0.540.56 (Fig. 3C; Supplemental Table 3C). In method 3 the correlation-based prereduction resulted in 49 features, which underwent PCA. The first 10 principal components explained about $80 \%$ of the variance (Supplemental Fig. 1). Twenty of the most important features were selected from them with AUCs of 0.57-0.59 (Fig. 3D; Supplemental Table 3D). The following 10 features were selected based on prior studies: entropy, joint entropy (GLCM), inverse different moment (GLCM), inverse difference moment normalized (GLCM), difference entropy (GLCM), sum average (GLCM), sum entropy (GLCM), high gray level run emphasis (GLRLM), short run emphasis (GLRLM), and zone percentage (GLSZM) (10,19-23). The multiple aggregation methods used in our dataset meant this translated to 52 features, which were reduced back to 10 by removing features highly correlated with entropy, resulting in AUCs of 0.60-0.62 (Fig. 3E; Supplemental Table 3E). Method 5 selected 17 features (Supplemental Fig. 2), and the 20 most important features are listed for method 6; both had AUCs of 0.62 (Figs. 3F-3G; Supplemental Fig. 3).

\section{DISCUSSION}

To the best of our knowledge, this is the first melanoma study to predict BRAFV600 mutation status with ${ }^{18} \mathrm{~F}-\mathrm{FDG}$ PET/CT features, while exploring several methods of feature selection in melanoma radiomics. Based on the driving effect of the BRAF mutation on the mitogen-activated protein kinase pathway and glucose metabolism in colorectal and thyroid cancer, with BRAF inhibitors in melanoma showing suppression of glycolysis and
${ }^{18} \mathrm{~F}$-FDG uptake, a mutation-based difference in ${ }^{18} \mathrm{~F}-\mathrm{FDG}$ uptake would be expected (30-32). However, no relationship between conventional PET features and BRAFV600 mutational status was found, either as an association or as a prediction. This result is, for most, inconsistent with prior research in thyroid and colorectal cancer, except for prior results in 2 thyroid cancer subtypes and 1 recent melanoma study $(31,33,34)$. Conventional $\mathrm{SUV}_{\max }$ predicts the BRAF mutation in thyroid cancer with an AUC of 0.75 and achieves a predictive accuracy of $75 \%$ in predicting KRAS/ BRAF status in colorectal cancer $(31,35)$. In Chang et al. (35), the AUC of the $\mathrm{SUV}_{\max }$-based prediction model for BRAF changed from 0.75 to 0.93 for lesions larger than $1 \mathrm{~cm}$, and to 0.64 for lesions smaller than $1 \mathrm{~cm}$. By including only lesions $2 \mathrm{~cm}$ or larger, we aimed to reduce such partial-volume effects, making their influence on our low predictive result less probable. In contrast, Hatt et al. (36) have proposed the inclusion of tumor volumes larger than $10 \mathrm{~cm}^{3}$ for radiomics research without excluding smaller lesions, instead reporting their correlation with volume. However, this study contained a variety of cancer types without melanoma patients and no metastatic disease. Tumor volumes larger than $10 \mathrm{~cm}^{3}$ in metastatic melanoma are rarer; therefore, our cutoff seems more practical and clinically relevant for this patient cohort, while taking the volume effect into account.

Radiomics features provide a more in-depth tumor characterization than purely ${ }^{18} \mathrm{~F}-\mathrm{FDG}$ uptake, such as tumor heterogeneity. Considering the influence of mutations on tumor phenotype, linking mutational status to the tumor phenotype provided by radiomics is the next logical step. Our AUCs varied from 0.54 to 0.62 , delivering low diagnostic predictive values. In models $1-3$, the confidence intervals included the value 0.50 , making them as valid as random chance. PET radiomics-based RAS mutation prediction in colorectal cancer shows AUCs of 0.65-0.79, and AUCs of 0.520.67 for epidermal growth factor receptor mutations in lung cancer, which is in line with our findings (5-7). According to sample size calculations for our study, we had a sufficient number of patients to significantly detect our predetermined clinically relevant 

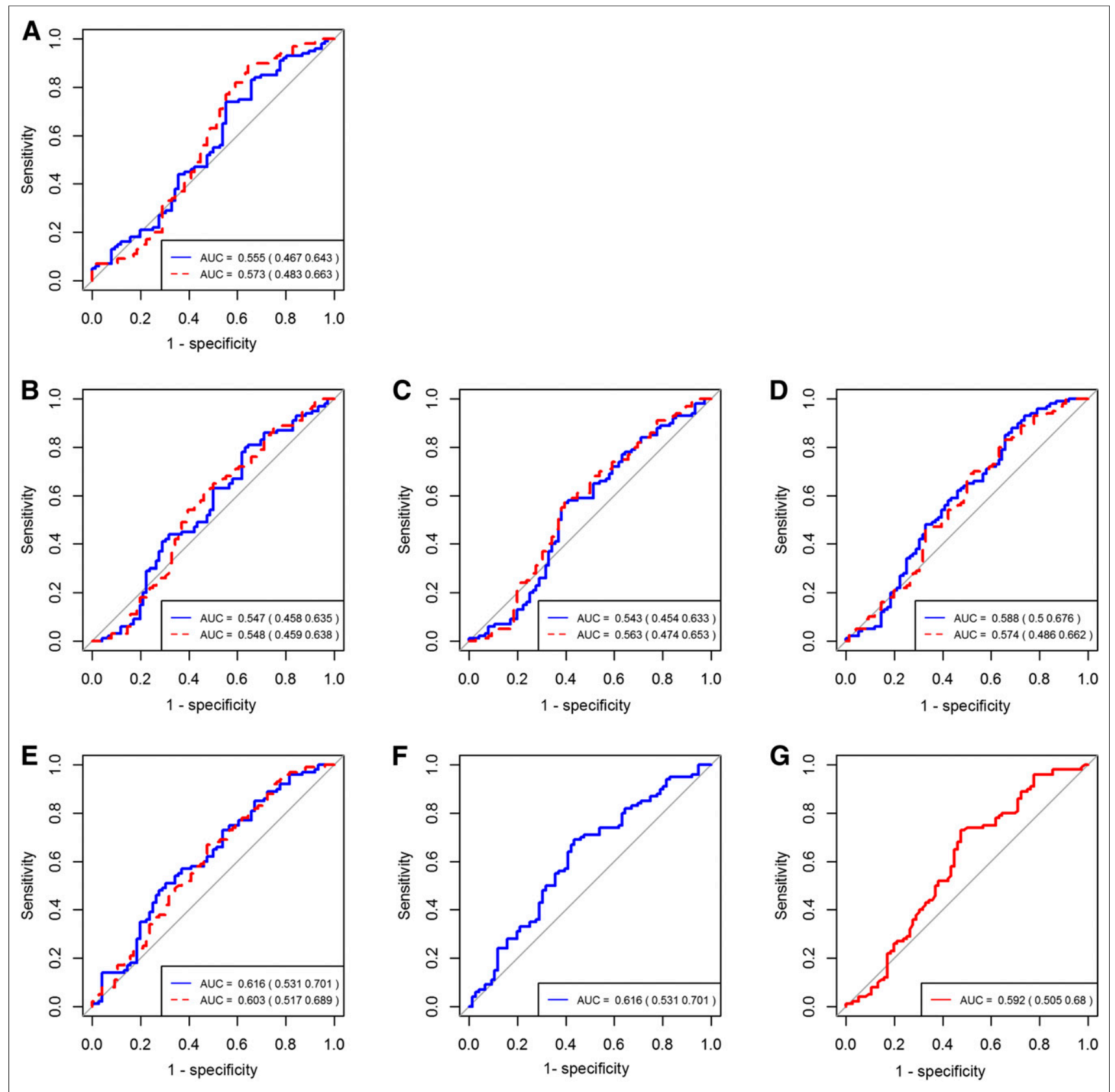

FIGURE 3. BRAFV600 prediction AUC. Shown are conventional PET features (A), method 1 (B), method 2 (C), method 3 (D), method 4 (E), method 5 $(F)$, and method $6(G)$, displayed with $95 \%$ confidence intervals in parentheses. Blue (straight) line = binary logistic regression; red (dashed) line $=$ RF.

effect in the conventional PET features analysis, which we did not find. As is often the case in radiomics, the sample size was modest for prediction model building, and future multicenter studies with standardized protocols could provide a solution for this. Based on our results, BRAF status in melanoma cannot yet be determined on ${ }^{18}$ F-FDG PET/CT scans alone and pathology remains the gold standard.

We explored several feature selection methods in melanoma radiomics. Highly correlated features essentially represent similar information and carry little individual added value when both are used in a predictive model: feature redundancy. In our first 3 methods, we used correlation matrices to reduce feature redundancy. Our results show that the extent of redundancy elimination varies dependent on the correlation coefficient cutoff chosen, use of subsequent PCA, and addition of conventional PET features. Raising the cutoff by 0.10 led to a $113 \%$ increase of selected features (49 vs. 23). Cutoffs of 0.90-0.95 have been used in PET radiomics studies in lung and vulvar cancer $(7,17)$. Interestingly adding conventional PET features $\mathrm{SUV}_{\text {peak }}$, MATV, and TLG did not reduce more variables and even added 1 more variable than expected, compared with method 1. We showed that the PCA provided the best feature reduction and highest AUC of these 3 methods.

In the final 3 methods we demonstrated a basic selection method and the yield of forgoing reduction. Previous studies have analyzed the radiomics robustness in several cancer types, providing a starting point for selection $(10,19-23)$. We chose often-mentioned 
robust features emphasizing good repeatability because multiple delineation methods were not analyzed. Considering the wide variability in choice, we focused on 10 features. Until more research has been done and consensus has been reached, this method is susceptible to the researchers' individual choices, making extrapolation difficult unless the same features are chosen. The simplest method of feature selection based on prior studies achieved the AUC value of 0.62 , which was similar to values achieved with the penalized binary logistic regression model and RF model, and higher than the other 3 models. Thus, highly advanced statistical methods do not always have the highest accuracy.

Our study has several limitations, the first being its retrospective nature, which hinders the standardization of PET protocols and reconstruction methods, another common problem in radiomics research. Overall, this effect was minimized in our study, since it was single-center and our scanners were cross-calibrated. In our study, 3 scans were found to be equal to the others except for the voxel matrix reconstruction. Since this influences radiomics feature calculation but not conventional PET measurements, these lesions were excluded from the radiomics analysis. Contrary to other studies, clinical predictors were not added to the prediction models. As often the case in radiomics, the sample size was modest for prediction model building, and future multicenter studies with standardized protocols could provide a solution for this. During cross-validation of the models, no separate external cohort was used, which would ensure a better extrapolation to the intended patient cohort. As this study shows, in PET melanoma radiomics research, as in all radiomics research, it is imperative to realize that the chosen feature selection method influences model performance and that researchers should make an informed decision on which method to choose. However, with our current knowledge, ${ }^{18} \mathrm{~F}$-FDG PET/CT-derived features alone do not yet adequately predict BRAF status in melanoma.

\section{CONCLUSION}

BRAFV600 mutation status is not associated with, nor can it be predicted with, conventional PET features, whereas radiomics features were of low predictive value $(\mathrm{AUC}=0.62)$. We showed feature selection methods to influence predictive model performance, describing and evaluating 6 unique methods. Detecting BRAFV600 status in melanoma based on ${ }^{18} \mathrm{~F}$-FDG PET/CT alone does not yet provide clinically relevant knowledge.

\section{DISCLOSURE}

Unrelated to this work John Haanen has provided consultation, attended advisory boards, and/or provided lectures for Pfizer, Bayer, MSD, BMS, IPSEN, Novartis, Roche/Genentech, Neon Therapeutics, Celsius Therapeutics, Gadeta BV, Immunocore, Seattle Genetics and AZ/MedImmune for which the Netherlands Cancer Institute received honoraria, and through his work the Netherlands Cancer Institute received grant support from BMS, MSD, GSK, Novartis and Neon Therapeutics. No other potential conflict of interest relevant to this article was reported.

\section{ACKNOWLEDGMENTS}

We thank Simone Pieplenbosch for her technical support and Katarzyna Jóźwiak for her additional statistical review.
KEY POINTS

QUESTION: Can conventional or radiomics-based ${ }^{18} \mathrm{~F}-\mathrm{FDG}$ PET/CT features predict BRAFV600 mutation in melanoma, and does radiomics feature selection influence this?

PERTINENT FINDINGS: This retrospective cohort study shows that BRAFV600 mutation status is not associated with, nor can it be predicted with, conventional PET features and that radiomics features were of low predictive value (AUC $=0.62$ ), after extraction of features from baseline ${ }^{18} \mathrm{~F}-\mathrm{FDG}$ PET/CT scans. Feature selection methods influenced model performance.

IMPLICATIONS FOR PATIENT CARE: Detecting BRAFV600 status in melanoma based on ${ }^{18} \mathrm{~F}-\mathrm{FDG}$ PET/CT alone does not yet provide clinically relevant knowledge.

\section{REFERENCES}

1. Bray F, Ferlay J, Soerjomataram I, Siegel RL, Torre LA, Jemal A. Global cancer statistics 2018: GLOBOCAN estimates of incidence and mortality worldwide for 36 cancers in 185 countries. CA Cancer J Clin. 2018;68: 394-424.

2. Wong ANMM, McArthur GA, Hofman MS, Hicks RJ. The advantages and challenges of using FDG PET/CT for response assessment in melanoma in the era of targeted agents and immunotherapy. Eur J Nucl Med Mol Imaging. 2017;44: 67-77.

3. Ascierto PA, Kirkwood JM, Grob JJ, et al. The role of BRAF V600 mutation in melanoma. J Transl Med. 2012;10:85.

4. Sachpekidis C, Anwar H, Winkler J, et al. The role of interim ${ }^{18} \mathrm{~F}-\mathrm{FDG}$ PET/CT in prediction of response to ipilimumab treatment in metastatic melanoma. Eur J Nucl Med Mol Imaging. 2018;45:1289-1296.

5. Chen SW, Shen WC, Chen WTL, et al. Metabolic imaging phenotype using radiomics of $\left[{ }^{18} \mathrm{~F}\right] \mathrm{FDG} \mathrm{PET} / \mathrm{CT}$ associated with genetic alterations of colorectal cancer. Mol Imaging Biol. 2019;21:183-190.

6. Lovinfosse P, Koopmansch B, Lambert F, et al. ${ }^{18}$ F-FDG PET/CT imaging in rectal cancer: relationship with the RAS mutational status. Br J Radiol. 2016;89: 20160212 .

7. Yip SSF, Kim J, Coroller TP, et al. Associations between somatic mutations and metabolic imaging phenotypes in non-small cell lung cancer. $\mathrm{J} \mathrm{Nucl} \mathrm{Med}$. 2017;58:569-576.

8. Keek SA, Leijenaar RT, Jochems A, Woodruff HC. A review on radiomics and the future of theranostics for patient selection in precision medicine. Br J Radiol. 2018;91:20170926

9. Wahl RL, Jacene H, Kasamon Y, Lodge MA. From RECIST to PERCIST: evolving considerations for PET response criteria in solid tumors. $J$ Nucl Med. 2009;50(suppl 1):122S-150S.

10. van Velden FHP, Kramer GM, Frings V, et al. Repeatability of radiomic features in non-small-cell lung cancer $\left[{ }^{18} \mathrm{~F}\right] \mathrm{FDG}-\mathrm{PET} / \mathrm{CT}$ studies: impact of reconstruction and delineation. Mol Imaging Biol. 2016;18:788-795.

11. Pfaehler E, Zwanenburg A, de Jong JR, Boellaard R. RACAT: an open source and easy to use radiomics calculator tool. PLoS One. 2019;14:e0212223.

12. Zwanenburg A, Leger S, Vallières $M$, Löck S. Image biomarker standardisation initiative. arXiv preprint arXiv:1612.07003. https://arxiv.org/abs/1612.07003. Updated May 16, 2019. Accessed January 2, 2019.

13. Boellaard R, Delgado-Bolton R, Oyen WJG, et al. FDG PET/CT: EANM procedure guidelines for tumour imaging: version 2.0. Eur J Nucl Med Mol Imaging. 2015;42:328-354.

14. Park SG, Lee JH, Lee WA, Han KM. Biologic correlation between glucose transporters, hexokinase-II, Ki-67 and FDG uptake in malignant melanoma. Nucl Med Biol. 2012;39:1167-1172.

15. de Langen AJ, Vincent A, Velasquez LM, et al. Repeatability of ${ }^{18}$ F-FDG uptake measurements in tumors: a metaanalysis. J Nucl Med. 2012;53:701-708.

16. Breiman L. Random forests. Mach Learn. 2001;45:5-32.

17. Collarino A, Garganese G, Fragomeni SM, et al. Radiomics in vulvar cancer: first clinical experience using ${ }^{18}$ F-FDG PET/CT images. J Nucl Med. 2019;60:199206.

18. Chalkidou A, O'Doherty MJ, Marsden PK. False discovery rates in PET and CT studies with texture features: a systematic review. PLoS One. 2015;10(suppl 5):e0124165.

19. Buvat I, Orlhac F, Soussan M. Tumor texture analysis in PET: where do we stand? J Nucl Med. 2015;56:1642-1644. 
20. Orlhac F, Soussan M, Maisonobe JA, Garcia CA, Vanderlinden B, Buvat I. Tumor texture analysis in ${ }^{18} \mathrm{~F}$-FDG PET: relationships between texture parameters, histogram indices, standardized uptake values, metabolic volumes, and total lesion glycolysis. J Nucl Med. 2014;55:414-422.

21. Leijenaar RTH, Carvalho S, Velazquez ER, et al. Stability of FDG-PET radiomics features: an integrated analysis of test-retest and inter-observer variability. Acta Oncol. 2013;52:1391-1397.

22. Bailly C, Bodet-Milin C, Couespel S, et al. Revisiting the robustness of PET-based textural features in the context of multi-centric trials. PLoS One. 2016;11:e0159984.

23. Yan J, Chu-Shern JL, Loi HY, et al. Impact of image reconstruction settings on texture features in ${ }^{18}$ F-FDG PET. J Nucl Med. 2015;56:1667-1673.

24. Zou H, Hastie T. Regularization and variable selection via the elastic net. J R Stat Soc Ser B. 2005;67:301-320.

25. Bates D, Mächler M, Bolker B, Walker S. Fitting linear mixed-effects models using lme4. J Stat Softw. 2015;67:251-264.

26. Pinheiro J, Bates D. DebRoy S, Sarkar D. nlme: linear and nonlinear mixed effects models. $R$ Dev Core Team. 2007;3:1-97.

27. Kuhn M, Wing J, Weston S, et al. Caret: classification and regression training. $R$ Package Version. 2018;6.0:81.

28. Liaw A, Wiener M. Classification and regression by randomforest. $R$ News. 2002;2:18-22.

29. Friedman J, Hastie T, Tibshirani R. Regularization paths for generalized linear models via coordinate descent. J Stat Softw. 2010;33:1-22.
30. Yun J, Rago C, Cheong I, et al. Glucose deprivation contributes to the development of KRAS pathway mutations in tumor cells. Science. 2009;325:15551559.

31. Kawada K, Nakamoto Y, Kawada M, et al. Relationship between ${ }^{18}$ F-fluorodeoxyglucose accumulation and KRAS/BRAF mutations in colorectal cancer. Clin Cancer Res. 2012;18:1696-1703.

32. McArthur GA, Puzanov I, Amaravadi R, et al. Marked, homogeneous, and early $\left[{ }^{18} \mathrm{~F}\right]$ fluorodeoxyglucose-positron emission tomography responses to vemurafenib in BRAF-mutant advanced melanoma. J Clin Oncol. 2012;30: $1628-1634$.

33. Santhanam P, Khthir R, Solnes LB, Ladenson PW. The relationship of BRAFV600E mutation status to FDG PET/CT avidity in thyroid cancer: a review and meta-analysis. Endocr Pract. 2018;24:21-26.

34. de Heer EC, Brouwers AH, Boellaard R, et al. Mapping heterogeneity in glucose uptake in metastatic melanoma using quantitative ${ }^{18}$ F-FDG PET/CT analysis. EJNMMI Res. 2018;8:101.

35. Chang JW, Park KW, Heo JH, et al. Relationship between ${ }^{18} \mathrm{~F}$-fluorodeoxyglucose accumulation and the BRAFV600E mutation in papillary thyroid cancer. World J Surg. 2018;42:114-122.

36. Hatt M, Majdoub M, Vallières M, et al. ${ }^{18}$ F-FDG PET uptake characterization through texture analysis: investigating the complementary nature of heterogeneity and functional tumor volume in a multi-cancer site patient cohort. $\mathrm{J} \mathrm{Nucl}$ Med. 2015;56:38-44. 